\title{
Influência da técnica de dispersão nas propriedades de sílica ativa
}

\section{(Influence of the dispersion process in the silica fume properties)}

\author{
R. C. O. Romano, H. Schreurs, V. M. John, R. G. Pileggi \\ Departamento de Construção Civil, Escola Politécnica, Universidade de S. Paulo - USP \\ Av. Prof. Almeida Prado, Trav. 2 n. 83, S. Paulo, SP 05424-970 \\ rcorjau@gmail.com,harryschreurs@gmail.com,vanderley.john@poli.usp.br,rafael.pileggi@poli.usp.br
}

\begin{abstract}
Resumo
A sílica ativa tem sido empregada em composições de materiais cimentícios com o intuito de aumentar a compacidade e/ou a resistência devido à sua atividade pozolânica e à sua atuação como filler promovida por sua granulometria muito fina. Contudo, para alcançar esses efeitos a sílica ativa deve se encontrar desaglomerada e dispersa, expondo assim toda a sua superfície reativa, além de possibilitar que suas partículas preencham os espaços vazios no empacotamento. No entanto, devido ao processo de densificação a que normalmente são submetidas, suas partículas se encontram fortemente unidas, podendo resultar, inclusive, em aglomerados macroscópicos que não se rompem com facilidade mesmo após cisalhamento intenso. Nesse caso, os benefícios esperados pelo uso da sílica ativa não são alcançados em sua plenitude. Desta forma, foram avaliadas as características de dispersão em água de duas sílicas ativas comerciais, as quais foram submetidas a condições de cisalhamento brando e intenso em um misturador convencional de baixa energia e em um dispersor de alta energia de cisalhamento, respectivamente.

Palavras-chave: sílica ativa, dispersão, energia de cisalhamento.
\end{abstract}

\begin{abstract}
Silica fume (SF) has been widely used in cement based products, as it promotes a better packing and increases its mechanical strength, either by its pozzolanic activity, and/or by the filler effect promoted by its fine particle size distribution. However, in order to achieve these benefits, the SF must be dispersed in the suspension, exposing completely its reactive surface and improving particle packing. As a by-product of metallic silicon production, SF particles are strongly bonded as a consequence of densification process involved during its generation, resulting in macroscopic agglomerated grains that require high energy for an effective dispersion. Then, the present study reports the SF dispersion in deionized water under low and high shear conditions, imposed by using a conventional laboratory suspension mixer and a high energy in-line mixer, respectively.
\end{abstract}

Keywords: silica fume, dispersion, shear energy.

\section{INTRODUÇ̃̃O}

A sílica ativa (SA) é uma matéria-prima muito fina composta por partículas com morfologia de esferas 'quase' perfeitas com diâmetros que podem variar de 0,02 a $0,5 \mu \mathrm{m}$ (com diâmetro médio de $0,1 \mu \mathrm{m}$ ) e área superficial em torno de $20 \mathrm{~m}^{2} / \mathrm{g}$ [1]. Tipicamente, de 85 a $95 \%$ de SA está na forma amorfa, com algumas impurezas, como silício metálico e carbono [2]. Em composições cimentícias, a SA tem sido utilizada para aumentar a resistência mecânica e compacidade devido, principalmente, à ocorrência de reações pozolânicas e ao efeito físico filler [3,4]. A primeira ocorre devido à interação com o hidróxido de cálcio do cimento, produzindo silicato de cálcio hidratado (C-S-H), material mais resistente e estável, que favorece a durabilidade dos compósitos, aumentando a resistência e diminuindo a permeabilidade do sistema devido ao processo de refinamento dos poros $[3,5]$. Já o efeito filler ocorre devido à diminuição da porosidade total do sistema promovida pelo preenchimento dos vazios de empacotamento, de poros capilares e de gel [6].
Usualmente, as SA têm sido comercializadas na forma densificada devido às facilidades de manuseio, transporte $\mathrm{e}$ armazenamento. Apesar dessas vantagens operacionais, foi demonstrado que as SA densificadas (grânulos $>100 \mu \mathrm{m}$ ) apresentam menor atividade pozolânica que as mesmas matérias-primas não-densificadas $(10-20 \mu \mathrm{m})$ [7].

Foi feita uma avaliação microestrutural e morfológica de aglomerados de SA presentes em pastas cimentícias (Fig. 1), tendo sido comprovado que as reações pozolânicas ocorrem predominantemente na superfície externa dos aglomerados, uma vez os mesmos apresentam centro vazio circundado por uma borda compacta de micro-partículas de SA [8].

O emprego de SA nessas condições não resulta na otimização dos benefícios potencialmente associados à adição dessa matéria-prima. Para evitar tal problema, uma alternativa tem sido a adição de SA na forma de dispersões coloidais previamente desaglomeradas, garantindo assim um ganho de desempenho nos materiais cimentícios [3,9]. Contudo, a sílica ativa não é comercializada desta forma em muitos países, inclusive no Brasil. 

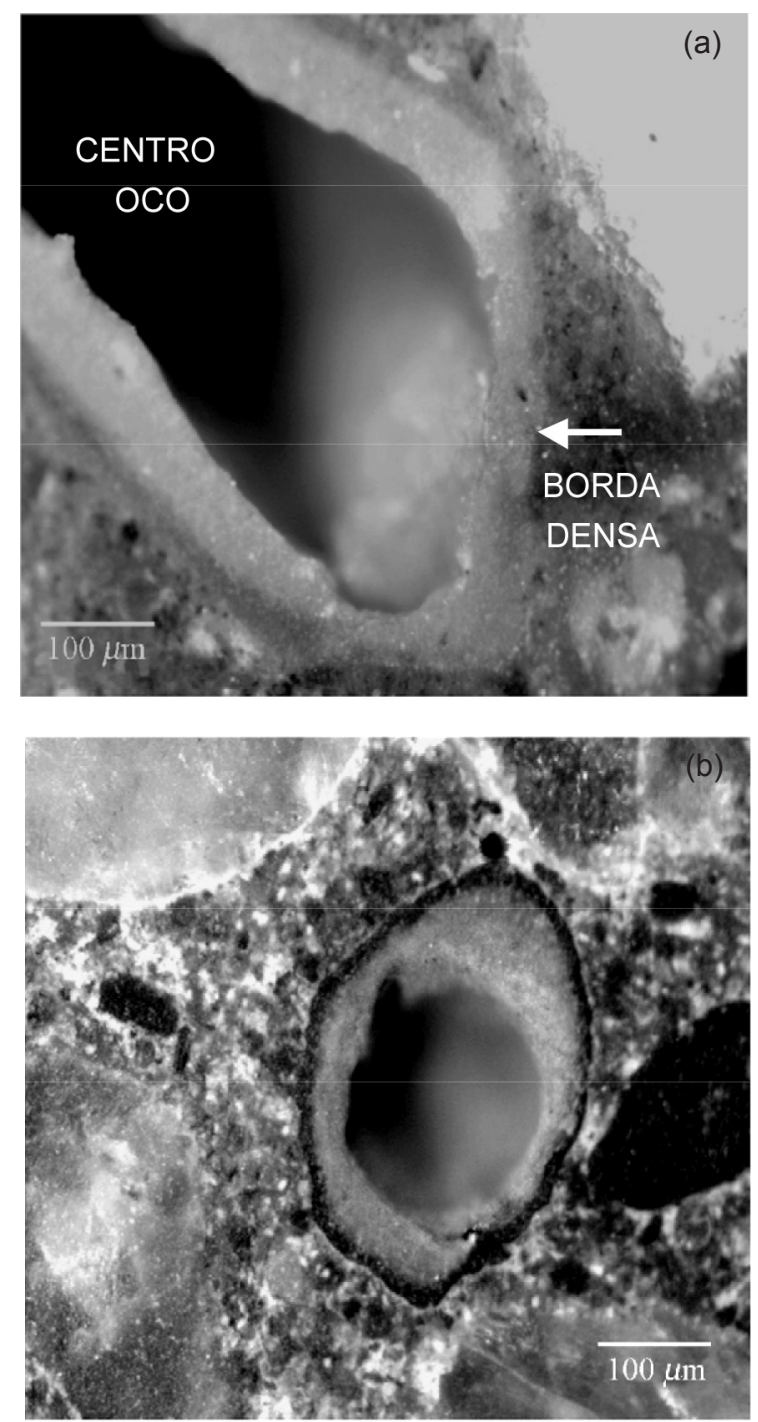

Figura 1: Microscopia ótica da microestrutura de uma argamassa contendo sílica ativa após 39 dias de imersão em $\mathrm{NaOH} 1 \mathrm{~N}$ e $80{ }^{\circ} \mathrm{C}[8]$.

[Figure 1: Optical micrographs of the mortar containing SF after 39 days [8]. ]

O presente trabalho tem como objetivo, portanto, avaliar a influência da condição de mistura nas características de dispersão em água de duas sílicas ativas comerciais, uma densificada e outra não densificada, as quais foram submetidas a condições de cisalhamento brando e intenso em um misturador convencional de baixa energia e em um dispersor de alta energia de cisalhamento, respectivamente. O trabalho propõe ainda uma metodologia simples para avaliação da eficiência de dispersão da sílica ativa, que pode ser utilizada em procedimentos de controle de qualidade em escala industrial.

\section{MATERIAIS E MÉTODOS}

A Tabela I apresenta as SA comerciais avaliadas neste estudo, descrevendo sua categoria, além de algumas características físicas, como área específica (BET, Gemini III2375, Micromeritics) e densidade aparente (picnômetro de hélio, MultiPycnometer, Quantachrome Instruments).

Tabela I - Características das SA densificada (D) e nãodensificada (ND) utilizadas no estudo.

[Table I - Densified (D) and non-densified (ND) SF properties]

\begin{tabular}{llc}
\hline Categoria & $\begin{array}{c}\text { Área superficial } \\
\left(\mathrm{m}^{2} / \mathrm{g}\right)\end{array}$ & $\begin{array}{c}\text { Densidade } \\
\left(\mathrm{g} / \mathrm{cm}^{3}\right)\end{array}$ \\
\hline Não densificada & 17,19 & $2,14 \pm 0,013$ \\
Densificada & 21,74 & $2,17 \pm 0,012$ \\
\hline
\end{tabular}

\section{Procedimento de mistura}

As suspensões de SA (teor de sólidos $50 \mathrm{~g} / \mathrm{L}$ ) foram processadas em dois misturadores diferentes, um rotacional com hélice naval e baixa rotação e o outro um dispersor de cisalhamento forçado de alta rotação (Fig. 2), sendo este último um equipamento projetado para fornecer elevadas energias de cisalhamento. A diferença de conceito entre ambos estabeleceu as duas condições de cisalhamento aplicadas nesse trabalho:

Baixa energia de cisalhamento (MR): as suspensões de SA com concentração de $50 \mathrm{~g} / \mathrm{L}$ foram preparadas em um misturador de bancada com hélice naval (IKA, Labortechnik RW20), ilustrado na Fig. 2a, na velocidade de 500 rpm, por diferentes tempos de mistura (5, 30 e 60 minutos). A SA foi adicionada à água destilada presente em um Becker com diâmetro de $60 \mathrm{~mm}$ com a hélice em rotação. Os tempos de mistura definidos são referentes ao período posterior à total inclusão da SA na água.

Alta energia de cisalhamento (DISP): o processo de mistura inicial das suspensões foi equivalente ao de baixa energia acima descrito. Contudo, após total inclusão da SA na água, as suspensões foram cisalhadas em um dispersor de alta energia de cisalhamento - in line mixer (IKA, Labortecknik T25 basic), por 5 min sob 17.500 rpm (Fig. 2b).

As suspensões resultantes das diferentes histórias de cisalhamento foram caracterizadas por ensaios de granulometria a laser, microscopia eletrônica de varredura e eficiência na dispersão, seguindo os procedimentos descritos a seguir.

Caracterização granulométrica a laser - alíquotas das suspensões foram diluídas a $1 \mathrm{~g} / \mathrm{L}$ para caracterização do tamanho de partículas em granulômetro laser, Mastersizer S,V.2.19, Malvern Instruments Ltd. (faixa de identificação: $50 \mathrm{~nm}-3500 \mu \mathrm{m})$.

Microscopia eletrônica de varredura - os pós foram secos em estufa com circulação de ar a $105{ }^{\circ} \mathrm{C}$ por 24 h. Os mesmos foram depositados sobre porta-amostra específico, sendo metalizados com platina sob vácuo. As imagens de microscopia eletrônica foram obtidas em microscópio eletrônico de varredura, LEO Stereoscan 440 acoplado ao espectrômetro de dispersão de energia. 

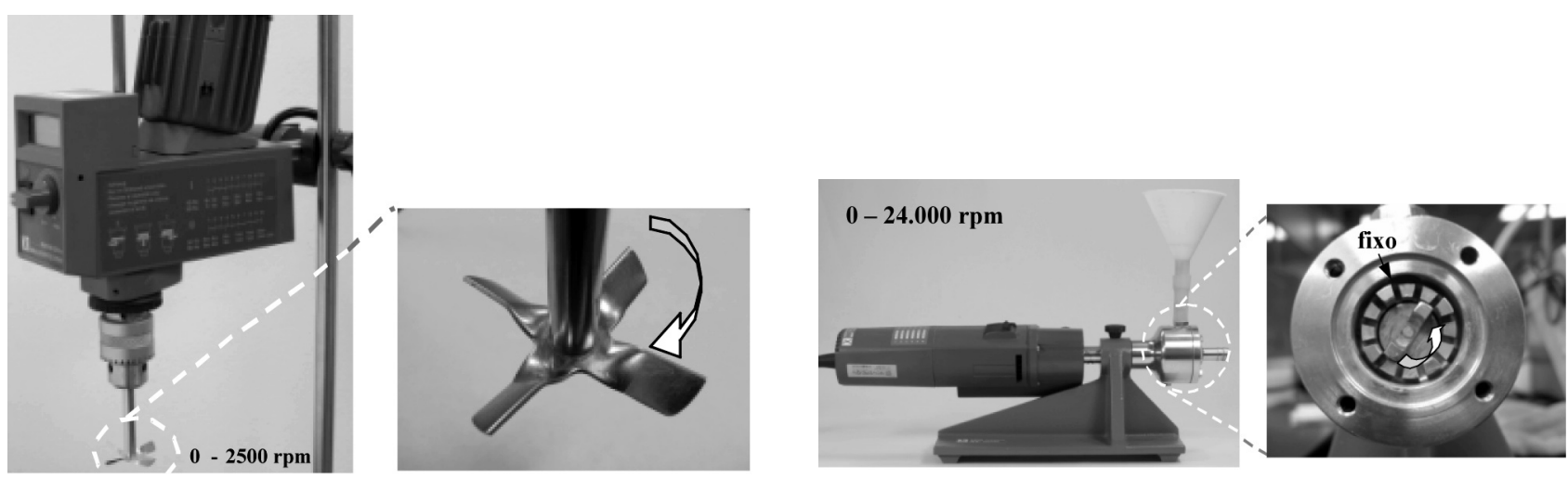

Figura 2: Equipamentos utilizados para dispersão da SA: (a) misturador rotacional com hélice naval IKA, modelo Labortechnik RW20, com potência do motor 70/35 W e rotação máxima $2.500 \mathrm{rpm}$; (b) dispersor de elevada energia de cisalhamento IKA modelo Labortechnik T25 basic com potência 500/300 W e rotação máxima $24.000 \mathrm{rpm}$.

[Figure 2: Mixers used for the SF dispersions: (a) rotational mixer with naval propeller (IKA, model Labortechnik RW20), maximum rotation 2500 rpm; (b) low shear energy in line mixer (IKA, model Labortechnik T25 basic), with maximum rotation 24.000 rpm.]

Eficiência na dispersão - amostras com volume aproximado $100 \mathrm{~mL}$ foram colocadas em repouso por 90 min em um béquer $(250 \mathrm{~mL})$. Após esse tempo, retirou-se o líquido sobrenadante com o auxílio de uma pipeta até atingir o material sedimentado, que foi seco em estufa a $105^{\circ} \mathrm{C}$ por $24 \mathrm{~h}$. A determinação da quantidade de partículas efetivamente dispersa, definida como eficiência na dispersão, foi calculada a partir das equações A e B a seguir,

$$
\begin{aligned}
& \mathrm{m}_{\mathrm{d}}=\mathrm{mi}-\mathrm{m}_{\mathrm{f}} \\
& \mathrm{e}_{\mathrm{d}}=\frac{\mathrm{md}}{\mathrm{m}_{\mathrm{i}}} \times 100 \%
\end{aligned}
$$

onde $m_{d}$ é a massa de silica ativa em suspensão (dispersa), $\mathrm{m}_{\mathrm{i}}$ é a massa total de silica ativa adicionada (inicial), $\mathrm{m}_{\mathrm{f}}$ é a massa seca de partículas sedimentadas e e é a eficiência na dispersão.

\section{RESULTADOS E DISCUSSÃO}

\section{Caracterização granulométrica e microscópica}

A Fig. 3 apresenta as distribuições granulométricas, discreta e acumulada, das sílicas ativas após serem processadas com diferentes histórias de cisalhamento. Em (a) são apresentadas as curvas granulométricas das SA processadas sob baixa energia de cisalhamento, enquanto que em (b) as curvas referentes à dispersão sob alta energia. A Tabela II apresenta os diâmetros característicos $\mathrm{D}_{10}, \mathrm{D}_{50}$ e $\mathrm{D}_{90}$.

A dificuldade de dispersão da sílica ativa em água é confirmada pela análise dos resultados da Fig. 3 e da Tabela II. Como observado, o emprego do misturador rotacional (MR) com menor energia de cisalhamento não resultou em melhoria significativa na dispersão, mesmo com aumento no tempo de mistura, uma vez que as curvas granulométricas obtidas para ambas SA foram semelhantes e com diâmetros elevados $\left(D_{50} \sim 45\right.$ a $\left.55 \mu \mathrm{m}\right)$. Por sua vez, o emprego do dispersor de alta energia de cisalhamento promoveu acentuada redução no diâmetro das partículas $\left(D_{50} \sim 20\right.$ a $25 \mu \mathrm{m})$.

O impacto do procedimento de mistura nas mudanças de granulometria pode ser numericamente estimado através da diferença entre as curvas granulométricas obtidas nas diferentes condições de mistura por uma curva referência (misturador rotacional 5 min - M5). Valores negativos observados nas curvas resultantes (Fig. 4) indicam redução na quantidade de partículas, enquanto valores positivos representam aumento na população de partículas nos diâmetros correspondentes.

A análise desses resultados evidencia o fato que o dispersor de cisalhamento intenso provocou uma sensível redução no diâmetro de grande parte do volume dos aglomerados na faixa de $100 \mu \mathrm{m}$, que foram reduzidos para aglomerado com diâmetros entre 20 a $30 \mu \mathrm{m}$. Esses resultados confirmam a maior eficiência deste equipamento em relação ao misturador rotacional, apesar de não ter sido possível desaglomerar as partículas primárias de SA (a faixa de diâmetros obtido é a limite permitida pelo equipamento). Pode-se assim inferir que há necessidade de utilização de equipamentos ainda mais eficientes para total desaglomeração da SA.

Quando se compara as distribuições granulométricas das SA somente em função da variação do processo de mistura (ou tempo de mistura), não se identificam diferenças relevantes. Sendo assim, é possível inferir que a SA não densificada utilizada neste estudo apresenta aglomerados com características semelhantes à densificada. Tal afirmação foi comprovada através da análise micrográfica de ambas SA (Fig. 5) processadas no misturador rotacional por 60 minutos, como visualizado nas Figs. 5A e 5B.

As Figs. 5C e 5D apresentam as micrografias das SA não densificada e densificada, respectivamente, após a mistura no dispersor de cisalhamento intenso. Como se 

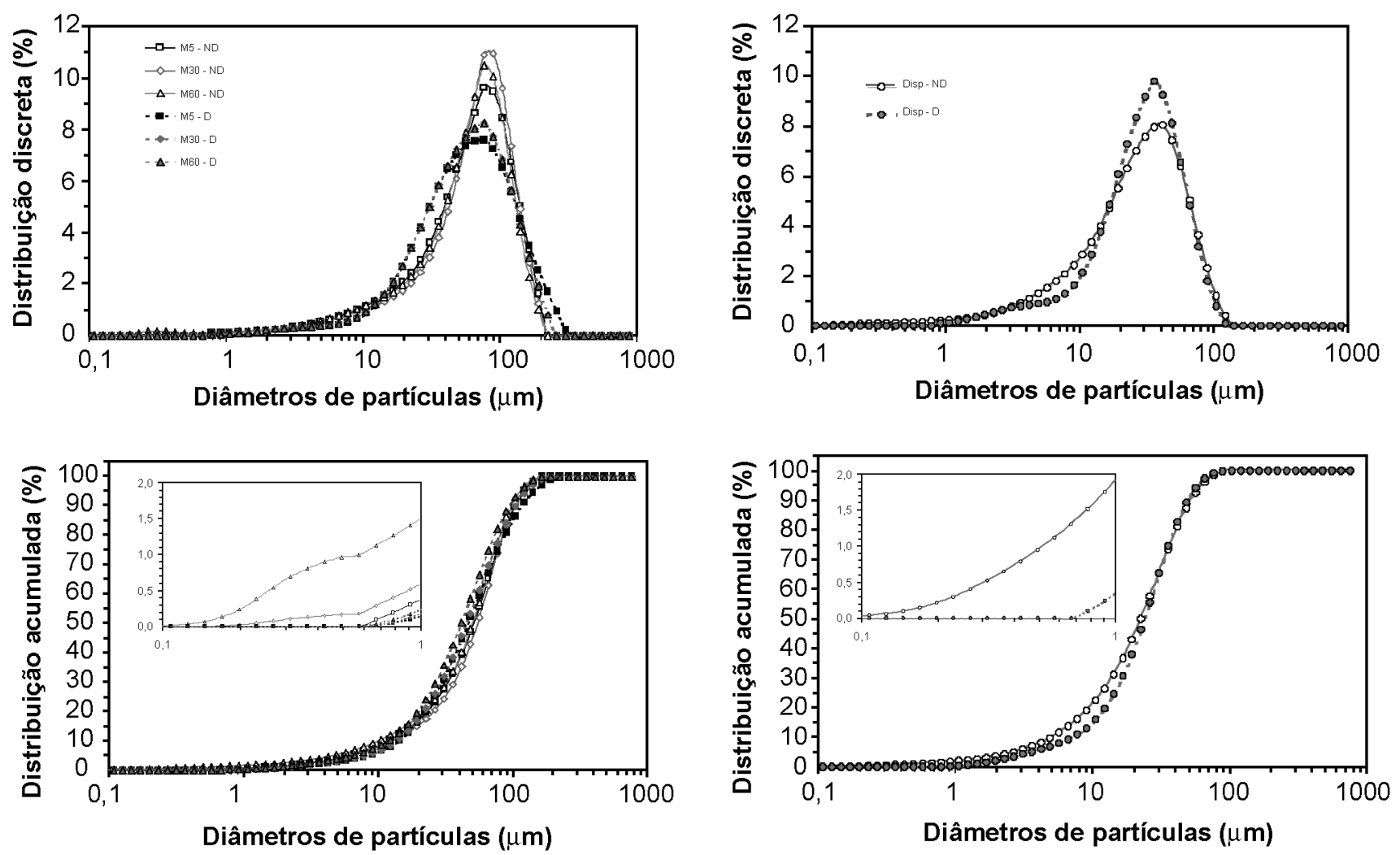

Figura 3: Comparação das granulometrias das SA processadas por diferentes tempos, sob baixa energia de cisalhamento (a) e sob alta energia de cisalhamento (b), destacando as porcentagens de partículas inferiores a 1,0 $\mu \mathrm{m}$ em ambos os casos, faixa de diâmetros que deveriam estar todas as partículas individualizadas.

[Figure 3: Comparison between SF granulometric distribution under low shear energy (a) and high shear energy (b), showing the particles percent shorter than $1.0 \mu \mathrm{m}$ for both cases.]

Tabela II - Valores de $\mathrm{D}_{10}, \mathrm{D}_{50}$ e $\mathrm{D}_{90}$ para as SA densificada e não densificada, em diferentes tempos e energias de agitação. [Table II - Values of $D_{10}, D_{50}$ e $D_{90}$ for densified and nondensified $S F$, in different times and mixing energies.]

\begin{tabular}{|c|c|c|c|c|c|c|c|c|}
\hline & \multicolumn{4}{|c|}{ Não densificada } & \multicolumn{4}{|c|}{ Densificada } \\
\hline & $\begin{array}{c}5 \\
\text { min }\end{array}$ & $\begin{array}{c}30 \\
\text { min } \\
\end{array}$ & $\begin{array}{c}60 \\
\text { min }\end{array}$ & IKA & $\begin{array}{c}5 \\
\min \end{array}$ & $\begin{array}{c}30 \\
\text { min }\end{array}$ & $\begin{array}{c}60 \\
\min \end{array}$ & IKA \\
\hline $\begin{array}{l}D_{10} \\
(\mu \mathrm{m})\end{array}$ & 12 & 12 & 10 & 5 & 15 & 15 & 12 & 8 \\
\hline $\begin{array}{c}\mathrm{D}_{50} \\
(\mu \mathrm{m})\end{array}$ & 50 & 55 & 50 & 25 & 45 & 45 & 40 & 20 \\
\hline $\begin{array}{c}\mathrm{D}_{90} \\
(\mu \mathrm{m})\end{array}$ & 104 & 101 & 96 & 55 & 116 & 104 & 94 & 50 \\
\hline
\end{tabular}

observa, apesar da maior capacidade de desaglomeração deste equipamento, os diâmetros finais dos aglomerados $(\sim$ $20 \mu \mathrm{m})$ são ainda muito maiores que as partículas individuais de SA $(\sim 0,1 \mu \mathrm{m})$. Essa avaliação reforça a observação de que é necessário um cisalhamento ainda mais intenso para se atingir maior eficiência na desaglomeração da SA.

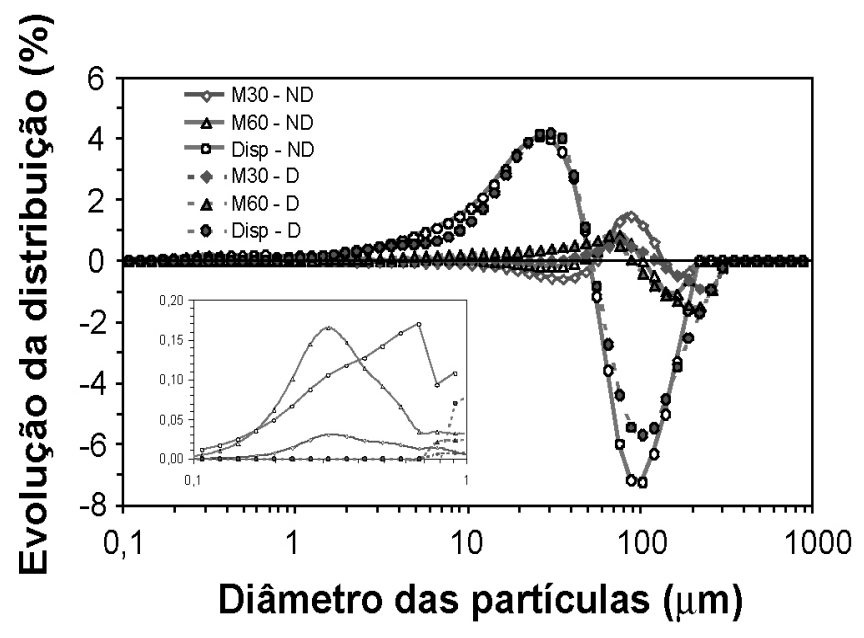

Figura 4: Evolução da distribuição granulométrica das SAs ND (linha contínua) e D (tracejado) em função de diferentes tempos e energias de mistura. O quadro em destaque apresenta o aumento da população de partículas com diâmetros entre 0,1 e 1,0 $\mu \mathrm{m}$.

[Figure 4: Evolution of the SF granulometric distribution. Nondesified $S F(N D)$ and densified $S F(D)$. The picture in focus shows the increase of the particle populations from 0.1 to $1.0 \mu \mathrm{m}$ diameters.] 

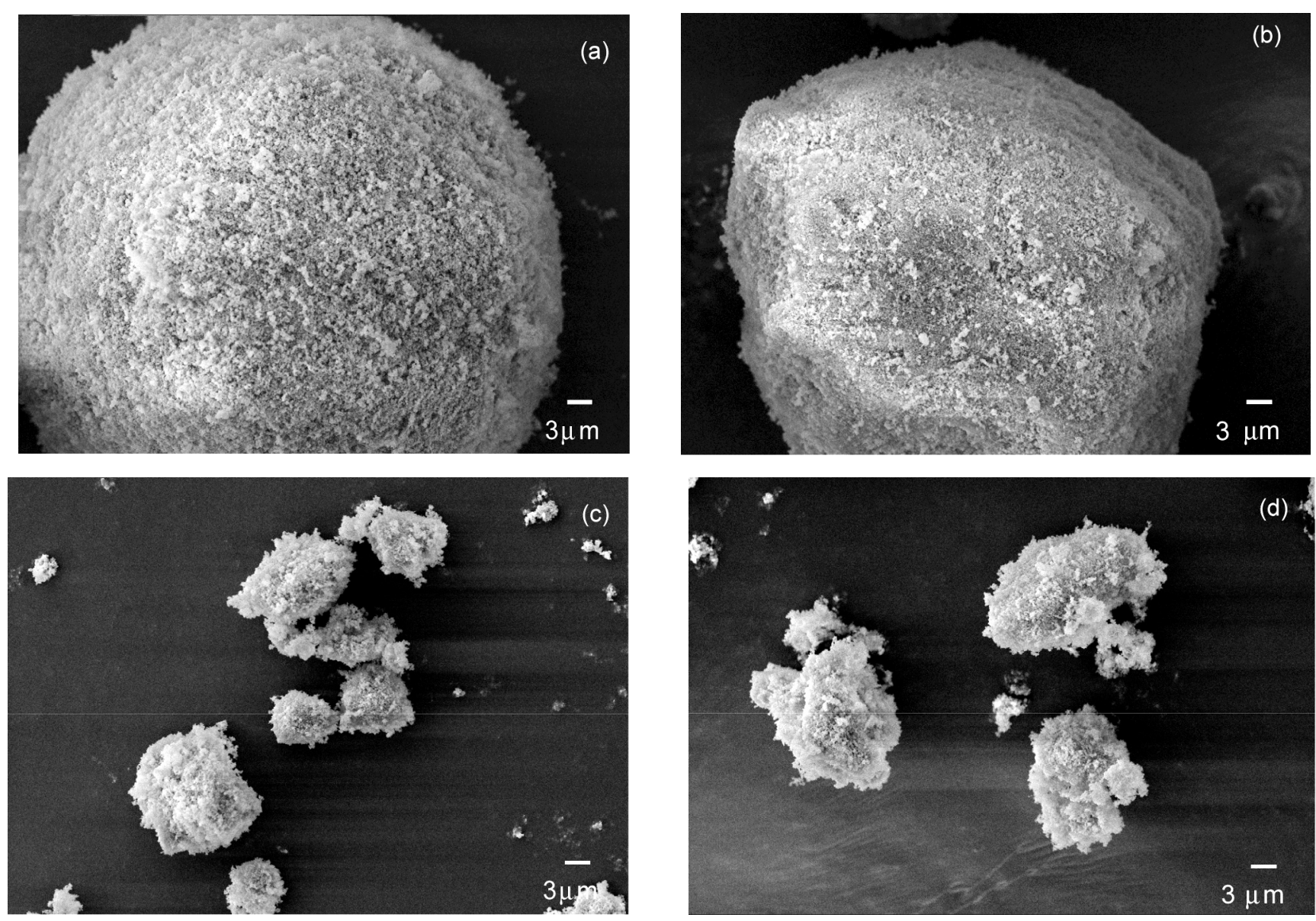

Figura 5: Microscopia eletrônica de varredura das sílicas ativas. As fotos representadas por A e C ilustram as variações dos diâmetros da SA não-densificada, para a mistura sob baixa energia (A) e alta energia de cisalhamento (C). Em B e D são ilustradas as mesmas alterações para a SA densificada, sendo que em B a foto foi tirada após processamento em baixa energia e em D após processamento em alta energia de cisalhamento.

[Figure 5: SEM micrographs of SF. The pictures $A$ and $C$ are the non-densified SF and $B$ and $D$ are the densified SF. A and B are the SEM after low shear energy processing and $C$ and $D$ are the high shear energy processing.]

\section{Eficiência na dispersão}

A quantificação da eficiência na dispersão da SA através de análises granulométricas e microscópicas é precisa. Contudo, esse procedimento apresenta elevado custo operacional e dificilmente poderia ser implementado como rotina em aplicações industriais. Uma alternativa simples para identificar o grau de desaglomeração da SA é através de ensaios de sedimentação, uma vez que diferenças granulométricas resultam em diferentes velocidades de precipitação das partículas e aglomerados em um meio líquido, segundo a lei de Stokes [10] descrita na equação C.

$$
v=\frac{1}{18 \eta} d^{2} \cdot g\left(\rho_{s}-\rho_{f}\right)
$$

A velocidade de sedimentação $v$ de uma partícula imersa em um meio líquido é diretamente proporcional ao quadrado de seu diâmetro $\mathrm{d}$, à aceleração da gravidade g e à diferença entre a densidade do sólido $\rho_{\mathrm{s}}$ e a densidade do meio líquido $\rho_{\mathrm{f}}$, mas é inversamente proporcional à viscosidade do fluido $\eta$ presente no sistema [10].

A eficiência na dispersão (Fig. 6) após repouso por 90 min das suspensões foi calculada pela equação B. Como se observa, o aumento no tempo de agitação no misturador rotacional provocou pequena elevação na quantidade de partículas em suspensão, enquanto o uso do dispersor incrementou significativamente a porcentagem de material que permaneceu em suspensão, atingindo o máximo de $62,7 \%$ para a SA não densificada.

Uma vez que a lei de Stokes descreve a ação da força da gravidade no processo de sedimentação de uma partícula, os resultados obtidos refletem diretamente as granulometrias e as micrografias previamente discutidas, ratificando a maior eficiência do dispersor. Contudo, os resultados de sedimentação apontam uma maior capacidade de manutenção de partículas em suspensão na SA não densificada nas diferentes condições de mistura, apesar das semelhanças granulométricas (Fig. 3) e de densidade entre as partículas de SA (Tabela I). 


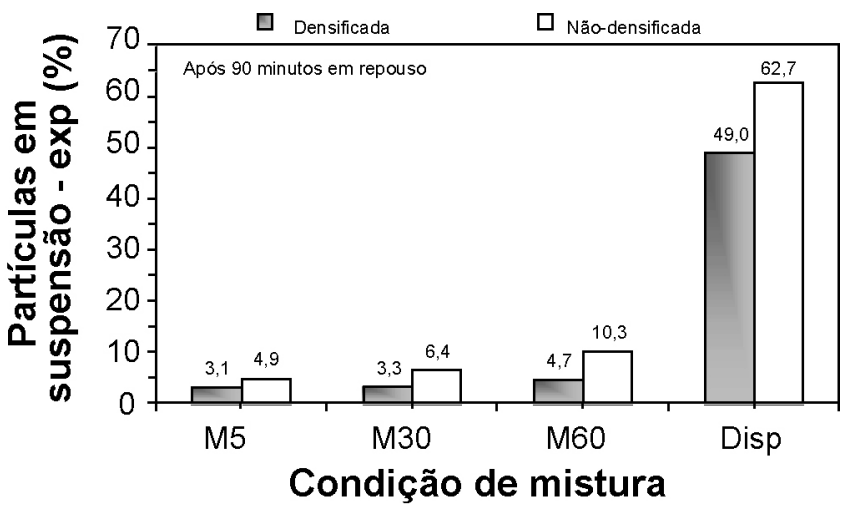

Figura 6: Quantidade de SA efetivamente dispersa após repouso de $90 \mathrm{~min}$.

[Figure 6: SF effectiveness dispersing after rest for 90 min.]

Considerando-se a lei de Stokes, uma causa provável deste resultado pode ser atribuída a uma menor densidade dos aglomerados da SA não densificada em relação aos da densificada. Tal hipótese é coerente com o processo de densificação das SA, que produz um pó mais denso e melhor compactado. Cabe ainda salientar que o aumento da população de partículas com menores diâmetros, resultado da variação do tempo de mistura e da dispersão sob alta energia de cisalhamento, não altera o $\mathrm{pH}$ da solução (permanecendo em torno de 8, distante do ponto isoelétrico da sílica ativa $(\mathrm{pH} \sim 2)$ ). Essa observação é importante, porque a alteração do $\mathrm{pH}$ poderia, também, afetar a dispersão das partículas. Assim, pode-se dizer que a maior eficiência na dispersão está diretamente relacionada com o aumento da população de partículas com menores diâmetros. Outro aspecto a ser discutido em relação à sedimentação, diz respeito à existência de um diâmetro crítico mínimo a partir do qual as partículas tendem a permanecer em suspensão devido ao movimento browniano de agitação térmica das moléculas do líquido [10]. Quanto maior o teor de finos, maior a barreira de partículas contra sedimentação. Sob esse aspecto, a SA não densificada apresentou teores ligeiramente maiores de partículas finas $(<10 \mu \mathrm{m})$ que a densificada, também contribuindo para sua menor velocidade de sedimentação.

\section{CONCLUSÕES}

Os resultados das caracterizações de granulometria, microscopia eletrônica de varredura e eficiência de dispersão atestam que o dispersor de elevada energia de cisalhamento foi mais eficiente que o misturador rotacional na dispersão da SA. Apesar disto, o dispersor utilizado permitiu a obtenção de partículas com diâmetro médio $\left(\mathrm{D}_{50}\right)$ de $20 \mu \mathrm{m}$, maior que o diâmetro das partículas primárias de $\mathrm{SA}$, as quais estão na faixa de $0,1 \mu \mathrm{m}$. Sendo assim, pode-se inferir que a total dispersão da SA demanda equipamentos ainda mais eficientes.
A utilização do ensaio de sedimentação mostrou-se uma ferramenta simples e capaz de avaliar a eficiência de dispersão das SA, sendo, portanto um método indicado para utilização em ambientes industriais.

Por fim, a SA não densificada apresentou menor tendência à sedimentação que a densificada. Esse resultado pode ser atribuído à presença de aglomerados menos densos e a um maior teor de partículas finas observadas nas diferentes condições de processo.

\section{AGRADECIMENTOS}

Os autores agradecem às empresas Infibra e Imbralit e ao CNPq e FINEP pelo apoio na realização deste trabalho.

\section{REFERÊNCIAS}

[1] R. D. Anjos, M. R. Ismael, F. T. Ramal Jr., V. C. Pandolfelli, "Adição de SA e a secagem de concretos refratários", Cerâmica 50 (2004) 331-335.

[2] D. R. G. Mitchell, I. Hinckzak, R. A. Day, "Interaction of silica fume with calcium hydroxide solutions and hydrated cement pastes", Cement Concrete Res. 28, 11 (1998) 1571 1584.

[3] P. C. Aitchin, Concreto de alto desempenho, $1^{\text {a }}$ Ed., Editora PINI, S. Paulo, SP (2000).

[4] J.F. De Larrard, J. F. Gorse, C. Push, "Comparative study of various silica fumes as additives in high-performance cementitious materials" Mater. and Structures 25 (1992) 265-272.

[5] M.A.S.Anjos, K. Ghavami, N.P. Barbosa, "Compósitos à base de cimento reforçado com polpa celulósica de bambu - Parte II - Uso de resíduos cerâmicos na matriz", Rev. Bras. Eng. Agr. Amb. 7, 2 (2003) 346-349.

[6] R. V. Velasco, "Concreto de Alto Desempenho Reforçado com Fibras de Polipropileno e Sisal Submetido a Altas Temperaturas",. Tese, UFRJ, COPPE , Rio de Janeiro, RJ (2002).

[7] M. I. Sanches de Rojas, J. Rivera, M. Frias, "Influence of the microsilica state on pozzolanic reaction rate", Cement Concrete Res. 29 (1999) 945-949.

[8] M.C. G. Juenger, C. P. Ostertag, "Alkali-silica reactivity of large silica fume-derived particles", Cement Concrete Res. 34 (2004) 1389-1402.

[9] M. S. Barata, "Concreto de alto desempenho no Estado do Pará: Estudo de viabilidade técnica e econômica de produção de concreto de alto desempenho com os materiais disponíveis em Belém através do emprego de adições de SA e metacaulim", Tese de Livre Docência, UFRGS, Porto Alegre, RS (1998).

[10] N. A. Mendonça, M. M. Oliveira,Apostila de Hidrologia urbana e drenagem, UFMG, Programa de Pós-Graduação em Saneamento, Meio Ambiente e Recursos Hídricos, Belo Horizonte , MG (2005) 10p.

(Rec.08/02/2008, Rev 14/02/2008, Ac. 17/02/2008) 